Publ. Math. Debrecen

87/3-4 (2015), 351-370

DOI: $10.5486 /$ PMD.2015.7197

\title{
Some new characterizations of Bloch type spaces of infinite matrices via Schur multipliers
}

\author{
By ANCA N. MARCOCI (Bucharest), LIVIU G. MARCOCI (Bucharest), \\ LARS-ERIK PERSSON (Luleå) and NICOLAE POPA (Bucharest)
}

\begin{abstract}
We consider the infinite matrix version $\mathcal{B}\left(D, \ell_{2}\right)$ of the Bloch space. In this paper we complement the results in the recent book [18] by deriving some new characterizations of $\mathcal{B}\left(D, \ell_{2}\right)$ and related spaces and duals via Schur multipliers. As applications we find the largest solid subspace of $\mathcal{B}\left(D, \ell_{2}\right)$ and its "conjugate" space $\mathcal{I}\left(\ell_{2}\right)$ considered in [18] and [19].
\end{abstract}

\section{Introduction}

It has been known for a long time that there is a formal relation between classical harmonic analysis and the theory of infinite matrices. Concerning this knowledge including historical remarks and applications we refer to the recent book [18] from 2014 by L. E. Persson and N. PopA and the references given there. However, still many challenging problems in this new theory of matricial harmonic analysis need to be investigated before we get a complete theory. The main aim of this paper is to give the solution to some of these new problems by presenting characterizations of Bloch type spaces of infinite matrices via Schur multipliers.

In Section 2 we discuss and give some properties of solid spaces of infinite matrices. One guiding observation for our investigations for this paper is the following: clearly the matrix spaces considered in our paper, $X$ are not solid spaces with respect to the order relation given by $A \geq 0$ if and only if $a_{i j} \geq 0$;

Mathematics Subject Classification: 15A60, 42B35, 43A99, 47B10.

Key words and phrases: Banach spaces of infinite matrices, Schur multipliers, upper triangular matrices, solid spaces. 
therefore the solid hull of these spaces $S(X)$, as well as their solid content $s(X)$, that is the smallest solid superspace, respectively the largest solid subspace of $X$, are of great interest.

In the case of function spaces $X$, the problem of finding $S(X)$ and $s(X)$, with respect to usual order relation on Taylor coefficients of the functions, is an old and difficult problem. For instance it follows from Littlewood's theorem on random power series [10, Theorem A.5, p. 228] that $S\left(H^{p}\right)=H^{2}, 2<p<\infty$, where $H^{p}$ is the usual Hardy space of order $p$. Much later, S. V. Kislyakov [11] showed that $S\left(H^{\infty}\right)=H^{2}$. Moreover, M. Jevtić and M. PAVLović [12] characterized $S\left(H^{p}\right), 0<p<1$ and $S\left(A^{1}\right)$, where $A^{1}$ is the Bergman space of order 1.

In $1976 \mathrm{~J}$. M. Anderson and A. Shields [2] found $s(\mathcal{B})$, where $\mathcal{B}$ is the Bloch space of analytic functions on the unit disk, and many other interesting results on the Taylor coefficients of functions from $\mathcal{B}$. Here the space of all coefficient multipliers from a space of analytic functions to a sequence space plays an important role.

For some matrix spaces $X$, the same problem was considered by F. LuSTPiquard [13], who showed that $S\left(B\left(\ell_{2}\right)\right)=\left\{A \mid A, A^{*} \in \ell_{\infty}\left(\ell_{2}\right)\right\}$. Here, of course, $B\left(\ell_{2}\right)$ is the space of all bounded operators (or matrices) on $\ell_{2}$, and $\ell_{\infty}\left(\ell_{2}\right)$ is the space of all infinite matrices $\left(a_{i j}\right)_{i, j \geq 1}$ such that

$\sup _{1<i}\left(\sum_{j=1}^{\infty}\left|a_{i j}\right|^{2}\right)^{1 / 2}<\infty$.

Some years later A. Pelczynski and F. Sukochev [16] showed that $s\left(B\left(\ell_{2}\right)\right)$ is the space of all Schur multipliers from the trace class $S_{1}$ into $\mathcal{M}_{1}$, the space of all infinite matrices with absolutely summable entries. The space of Schur multipliers is quite similar with the space of coefficient multipliers used by J. M. ANDER SON and A. SHIELDS [2].

In our paper we extend to matrix spaces some results from [2] and some of their techniques, especially in connection with the Abel duality. Moreover we characterize in terms of Schur multipliers $s\left(S_{q}\right), 1<q<\infty, S_{q}$ being the Schatten class of order $q$.

For two infinite matrices $A=\left(a_{j k}\right)$ and $B=\left(b_{j k}\right)$ their Schur product (or Hadamard product) is defined to be the matrix of elementwise products:

$$
A * B:=\left(a_{j k} b_{j k}\right) .
$$

If $X$ and $Y$ are two linear spaces of infinite matrices the space of Schur multipliers from $X$ to $Y$ is the space

$$
(X, Y):=\{M: M * A \in Y \text { for every } A \in X\},
$$


If $X$ and $Y$ are Banach spaces, then we consider on the space $(X, Y)$ the natural norm

$$
\|M\|_{(X, Y)}:=\sup _{\|A\|_{X} \leq 1}\|M * A\|_{Y} .
$$

Motivated by the papers [16] and [2] we investigate Schur multipliers on various spaces of infinite matrices.

We recall the matrix versions of Bloch and Bergman spaces (see e.g. [19], [14] and [18]). For a treatment of a more general case of Bloch and Bergman spaces in the case of vector valued functions we refer to [3], [6] and [7].

For an infinite matrix $A=\left(a_{i j}\right)$, and an integer $k$ we call the $k$ th-diagonal matrix associated to $A$ (see also [4] and [5]), denoted by $A_{k}$, the matrix whose entries $a_{i j}^{\prime}$ are given by

$$
a_{i j}^{\prime}=\left\{\begin{array}{l}
a_{i j} \text { if } j-i=k \\
0 \text { otherwise. }
\end{array}\right.
$$

We recall that for an upper triangular infinite matrix $A$, we define $A(r)$ as follows:

$$
A(r):=A * C(r),
$$

where $C(r)$ is the Toeplitz matrix associated with the Cauchy kernel $\frac{1}{1-r}$, for $0 \leq r<1$, that is

$$
C(r)=\left(\begin{array}{cccc}
1 & r & r^{2} & \ldots \\
0 & 1 & r & \ldots \\
0 & 0 & 1 & \ldots \\
\vdots & \vdots & \ddots & \ddots
\end{array}\right) .
$$

Let $D$ be the unit disk in $\mathbb{C}$. The matrix version of the Bloch space, denoted by $\mathcal{B}\left(D, \ell_{2}\right)$, consists of all upper triangular matrices $A$ such that

$$
\|A\|_{\mathcal{B}\left(D, \ell_{2}\right)}=\sup _{0 \leq r<1}\left(1-r^{2}\right)\left\|A^{\prime}(r)\right\|_{B\left(\ell_{2}\right)}+\left\|A_{0}\right\|_{B\left(\ell_{2}\right)}<\infty,
$$

where $B\left(\ell_{2}\right)$ is the usual operator norm of the matrix $A$ on the sequence space $\ell_{2}$ and $A^{\prime}(r)=\sum_{k=0}^{\infty} A_{k} k r^{k-1}$.

This space is interesting because it appears as the space of all Schur multipliers from some matrix versions of the classical spaces of analytic functions $H^{1}$ and $B M O A$, extending in the framework of infinite matrices a very nice theorem of M. Mateljević and M. Pavlović [15].

The space $\mathcal{B}_{0}\left(D, \ell_{2}\right)$ is the space of all upper triangular infinite matrices $A$ such that $\lim _{r \rightarrow 1^{-}}\left(1-r^{2}\right)\left\|(A * C(r))^{\prime}\right\|_{B\left(\ell_{2}\right)}=0$. 
354 Anca N. Marcoci, Liviu G. Marcoci, Lars-Erik Persson and Nicolae Popa

We denote with $S_{p}, 1 \leq p<\infty$, the Schatten class of order $p$, the space of all compact operators $T$ with its singular value sequence $\left\{\lambda_{n}\right\} \in \ell_{p}$ (see e.g. [21]).

The Bergman-Schatten space is defined by

where

$$
L_{a}^{1}\left(D, \ell_{2}\right):=\left\{\mathrm{A} \text { upper triangular matrix : }\|A\|_{L_{a}^{1}\left(D, \ell_{2}\right)}<\infty\right\}
$$

$$
\|A\|_{L_{a}^{1}\left(D, \ell_{2}\right)}:=\int_{0}^{1}\|A(r)\|_{S_{1}} 2 r d r .
$$

The paper is organized as follows: In the second Section we discuss the solid spaces for general spaces of infinite matrices and in particular we extend a result from [16], characterizing the Schur multipliers from the Schatten class of order $p$, $1<p<\infty$, to the space of matrices with absolute summable entries (see Theorem 2.3). Section 3 contains the main results, especially Theorems 3.3 and 3.5, and it is devoted to the study of Schur multipliers between the spaces $\mathcal{I}\left(\ell_{2}\right)$, $\mathcal{B}\left(D, \ell_{2}\right)$ and $\mathcal{A}\left(\ell_{2}\right)$, as well as some duality results. Finally, in the last Section we give some applications of our results regarding the largest solid subspaces of $\mathcal{B}\left(D, \ell_{2}\right)$ and $\mathcal{I}\left(\ell_{2}\right)$ as well as Schur multipliers from an arbitrary space of infinite matrices $X$ in these spaces.

\section{Solid spaces of infinite matrices}

For a matrix $A=\left(a_{i j}\right)$ the corresponding matrix of absolute values is defined by $A^{\#}:=\left(\left|a_{i j}\right|\right)$, and for a space of infinite matrices $X$ we denote by $X^{\#}$ the space of all infinite matrices $A$ such that $A^{\#} \in X$ (see e.g. [16]).

A space of infinite matrices $X$ is said to be solid, if the condition $A=\left(a_{i j}\right) \in X$ implies that $B=\left(b_{i j}\right) \in X$, for all matrices $B$ with $\left|b_{i j}\right| \leq\left|a_{i j}\right|, i, j \geq 1$. Equivalently, $X$ is solid if

$$
\mathcal{M}_{\infty} \subset(X, X)
$$

where $\mathcal{M}_{\infty}$ is the space of all infinite matrices with uniformly bounded entries, equipped with the natural norm

$$
\|A\|_{\mathcal{M}_{\infty}}=\sup _{i, j \geq 1}\left|a_{i j}\right|
$$

Next we present some elementary properties for solid spaces of infinite matrices.

Proposition 2.1. Let $X$ and $Y$ be Banach spaces of infinite matrices and $X$ or $Y$ be solid. Then the space of all Schur multipliers $(X, Y)$ from $X$ into $Y$ is also a solid space. 
Proof. Let $A \in \mathcal{M}_{\infty}$ and $B \in(X, Y)$. Assume that $Y$ is a solid space. For $C \in X$ we observe that, since $Y$ is solid, we get

$$
(A * B) * C=A *(B * C) \in Y \text {. }
$$

Hence, $(X, Y)$ is a solid space. The proof of the case when $X$ is assumed to be a solid space is similar so the proof is complete.

A similar result as in the case of sequences (see [2]) also holds for infinite matrices.

Proposition 2.2. Let $X$ be a Banach space of infinite matrices. Then there exists the largest solid subspace of infinite matrices $s(X) \subset X$. Moreover, we have that

with its natural norm.

$$
s(X)=\left(\mathcal{M}_{\infty}, X\right),
$$

Proof. Let us take $s(X)=\left(\mathcal{M}_{\infty}, X\right)$. Then it is clear from Proposition 2.1 that $s(X)$ is a solid subspace of $X$. If $Y$ is another solid space with $Y \subset X$, then we have that

$$
A * B \in Y \subset X,
$$

for every $A \in Y$ and $B \in \mathcal{M}_{\infty}$. It follows that

$$
A \in\left(\mathcal{M}_{\infty}, X\right)=s(X),
$$

and thus $s(X)$ is the largest solid subspace of $X$ with

$$
s(X)=\left(\mathcal{M}_{\infty}, X\right) .
$$

A. Pelczynski and F. Sukochev described in [16] the space of Schur multipliers $\left(B\left(\ell_{2}\right), \mathcal{M}_{1}\right)$, which maps the space of all bounded operators on $\ell_{2}$, namely $B\left(\ell_{2}\right)$, into matrices with absolutely summable entries, $\mathcal{M}_{1}$, where

$$
\mathcal{M}_{1}:=\left\{A \text { infinite matrix }: A=\left(a_{i j}\right)_{i, j \geq 0} \text { such that }\|A\|_{\mathcal{M}_{1}}=\sum_{i, j}\left|a_{i j}\right|<\infty\right\},
$$

and also the space of Schur multipliers $\left(S_{1}, \mathcal{M}_{1}\right)$, where $S_{1}$ is the Schatten class of order 1 (the trace class). More precisely, in [16] the authors proved the following identities:

(i) $\left(B\left(\ell_{2}\right), \mathcal{M}_{1}\right)=S_{1}^{\#}$;

(ii) $\left(S_{1}, \mathcal{M}_{1}\right)=B\left(\ell_{2}\right)^{\#}$. 
Let it call a matrix $A$ a regular matrix if $A=A_{1}-A_{2}$, where $A_{1}, A_{2} \in B\left(\ell_{2}\right)$, $A_{1} \geq 0, A_{2} \geq 0$.

We remark that $B\left(\ell_{2}\right)^{\#}=s\left(B\left(\ell_{2}\right)\right)$ is the largest solid subspace of $B\left(\ell_{2}\right)$, or, equivalently, is the subspace of all regular matrices from $B\left(\ell_{2}\right)$, endowed with the norm

$$
\|A\|_{B\left(\ell_{2}\right) \#}=\left\|A^{\#}\right\|_{B\left(\ell_{2}\right)}=\sup _{\substack{\mid t_{i j} j \leq 1 \\ i, j \geq 1}}\|A * T\|_{B\left(\ell_{2}\right)},
$$

and $S_{1}^{\#}=s\left(S_{1}\right)$ is equipped with the norm

$$
\|A\|_{S_{1}^{\#}}=\left\|A^{\#}\right\|_{S_{1}}=\sup _{\substack{\left|t_{i j}\right| \leq 1 \\ i, j \geq 1}}\|T * A\|_{S_{1}} .
$$

Our result is an extension of the equalities (i) and (ii) and characterizes the space of Schur multipliers which map the space $S_{p}$, the Schatten class of order $p$, $1<p<\infty$, into matrices with absolutely summable entries:

Theorem 2.3. Let $1<p<\infty$ and $1 / p+1 / q=1$.

Then

$$
\left(S_{p}, \mathcal{M}_{1}\right)=s\left(S_{q}\right)=\left(\mathcal{M}_{\infty}, S_{q}\right)
$$

with equality of the corresponding norms.

Proof. Let $A \in\left(S_{p}, \mathcal{M}_{1}\right)$. We denote by $\epsilon=\left(\epsilon_{j k}\right)$ the infinite matrix with $\left|\epsilon_{j k}\right| \leq 1$ for all $j, k \geq 1$. Then we have that

$$
\begin{aligned}
\|A\|_{\left(S_{p}, \mathcal{M}_{1}\right)} & =\sup _{\|B\|_{S_{p}} \leq 1} \sum_{j, k}\left|a_{j k} b_{j k}\right|=\sup _{\|B\|_{S_{p}} \leq 1} \sup _{\substack{\epsilon_{j k} \mid \leq 1 \\
j, k \geq 1}}\left|\sum_{j, k} \epsilon_{j k} a_{j k} b_{j k}\right| \\
& =\sup _{\substack{\left|\epsilon_{j k}\right| \leq 1 \\
j, k \geq 1}} \sup _{\|B\|_{S_{p}} \leq 1}\left|\operatorname{Tr}(\epsilon * A)^{t} B\right|=\sup _{\substack{\left|\epsilon_{j k}\right| \leq 1 \\
j, k \geq 1}}\left\|(\epsilon * A)^{t}\right\|_{S_{q}} \\
& =\sup _{\substack{\left|\epsilon_{j k}\right| \leq 1 \\
j, k \geq 1}}\|\epsilon * A\|_{S_{q}}=\|A\|_{S_{q}^{\#}}=\|A\|_{\left(\mathcal{M}_{\infty}, S_{q}\right)},
\end{aligned}
$$

where $\operatorname{Tr}(\cdot)$ denotes the trace of a matrix. The proof is complete.

\section{The main results}

In this Section we describe the upper triangular Schur multipliers acting on spaces of upper triangular infinite matrices (sometimes called analytic matrices, 
see e.g. the book [18, p. 2]). In the sequel we use another notation, which is more appropriate for our aims. For the entries of the matrix $A$, we put

$$
a_{k}^{l}=\left\{\begin{array}{l}
a_{l, l+k}, \quad k \geq 0, \quad l=1,2,3 \ldots, \\
a_{l-k, l}, \quad k<0, \quad l=1,2,3 \ldots
\end{array}\right.
$$

and denote $A$ by $A=\left(a_{k}^{l}\right)_{k \in \mathbb{Z}, l \geq 1}$ and in the case of upper triangular matrices $A=\left(a_{k}^{l}\right)_{k \geq 0, l \geq 1}$.

The first theorem asserts that all infinite matrices from $\mathcal{B}\left(D, \ell_{2}\right)$ are precisely all upper triangular Schur multipliers from $L_{a}^{1}\left(D, \ell_{2}\right)$ to $\mathcal{A}^{\prime}\left(\ell_{2}\right)$, where we denote by $\mathcal{A}^{\prime}\left(\ell_{2}\right)$ the space of all upper triangular matrices $A$, such that the diagonal sequences, denoted by $\left(a_{k}^{l}\right)_{l \geq 1}$, for $k \geq 0$, satisfy the following conditions:

(1) $\sup _{k \geq 0} \sum_{l=1}^{\infty}\left|a_{k}^{l}\right|<\infty$;

(2) there exists $\lim _{r \rightarrow 1^{-}} \sum_{k=0}^{\infty} \frac{r^{k}}{k+1} \sum_{l=1}^{\infty} a_{k}^{l}$.

Remark. By using the uniform boundedness principle conditions (1) and (2) imply

$$
\||A|\|:=\sup _{\substack{0 \leq r<1 \\\|B\|_{L_{a}^{1}\left(D, \ell_{2}\right)} \leq 1}}\left|\sum_{k=0}^{\infty} \frac{r^{k}}{k+1} \sum_{l=1}^{\infty} a_{k}^{l} b_{k}^{l}\right|<\infty .
$$

It is easy to see that $\||A|\|$ is a norm on the space of all upper triangular Schur multipliers $\mathcal{U T}\left(L_{a}^{1}\left(D, \ell_{2}\right), \mathcal{A}^{\prime}\left(\ell_{2}\right)\right)$.

Our first result of this Section reads:

Theorem 3.1. Let $\mathcal{U} \mathcal{T}\left(L_{a}^{1}\left(D, \ell_{2}\right), \mathcal{A}^{\prime}\left(\ell_{2}\right)\right)$ be the space of upper triangular Schur multipliers. Then we have that

as linear spaces.

$$
\mathcal{U} \mathcal{T}\left(L_{a}^{1}\left(D, \ell_{2}\right), \mathcal{A}^{\prime}\left(\ell_{2}\right)\right)=\mathcal{B}\left(D, \ell_{2}\right)
$$

Proof. Let $0 \leq r<1$. In [14] (see also [18], Theorem 7.11) it is proved that $\mathcal{B}\left(D, \ell_{2}\right)=L_{a}^{1}\left(D, \ell_{2}\right)^{*}$ by using the duality pair

$$
\langle A, B\rangle=\int_{0}^{1} \operatorname{Tr}\left[A(r) B^{*}(r)\right] 2 r d r=\int_{0}^{1} 2 \sum_{k=0}^{\infty} r^{2 k+1}\left(\sum_{l=1}^{\infty} a_{k}^{l} \overline{b_{k}^{l}}\right) d r
$$

where $A=\left(a_{k}^{l}\right)_{k \geq 0, l \geq 1} \in L_{a}^{1}\left(D, \ell_{2}\right), B=\left(b_{k}^{l}\right)_{k \geq 0, l \geq 1} \in \mathcal{B}\left(D, \ell_{2}\right)$, and

$$
|\langle A, B\rangle| \leq C\|A\|_{L_{a}^{1}\left(D, \ell_{2}\right)} \cdot\|B\|_{\mathcal{B}\left(D, \ell_{2}\right)}
$$


358 Anca N. Marcoci, Liviu G. Marcoci, Lars-Erik Persson and Nicolae Popa

for an absolute constant $C>0$.

We first prove that $\mathcal{B}\left(D, \ell_{2}\right) \subset \mathcal{U T}\left(L_{a}^{1}\left(D, \ell_{2}\right), \mathcal{A}^{\prime}\left(\ell_{2}\right)\right)$. We observe that

and

$$
\sup _{k \geq 0} \frac{2}{k+1} \sum_{l=1}^{\infty}\left|a_{k}^{l}\right| \leq\|A\|_{L_{a}^{1}\left(D, \ell_{2}\right)}
$$

$$
\sup _{k \geq 0, l \geq 1}\left|b_{k}^{l}\right| \leq\|B\|_{\mathcal{B}\left(D, \ell_{2}\right)}
$$

Then it follows

$$
\sup _{k \geq 0} \frac{2}{k+1} \sum_{l=1}^{\infty}\left|a_{k}^{l}\left\|b_{k}^{l} \mid \leq\right\| A\left\|_{L_{a}^{1}\left(D, \ell_{2}\right)} \cdot\right\| B \|_{\mathcal{B}\left(D, \ell_{2}\right)}\right.
$$

for all $k$, consequently the condition (1) is satisfied.

Now let $0<\rho<1$. Then we have that

$$
\begin{aligned}
|\langle A(\rho), B\rangle| & =\left|\int_{0}^{1}\left[\sum_{k=0}^{\infty} \rho^{k} r^{2 k}\left(\sum_{l=1}^{\infty} a_{k}^{l} \overline{b_{k}^{l}}\right)\right](2 r d r)\right| \\
& \leq \int_{0}^{1} \sum_{k=0}^{\infty} \rho^{k} r^{2 k}\left(\sum_{l=1}^{\infty}\left|a_{k}^{l}\right|\left|b_{k}^{l}\right|\right) 2 r d r \\
& \leq \int_{0}^{1}\left(\sum_{k=0}^{\infty}(k+1) \rho^{k} r^{2 k}\right) \cdot\|A\|_{L_{a}^{1}\left(D, \ell_{2}\right)} \cdot\|B\|_{\mathcal{B}\left(D, \ell_{2}\right)} r d r \\
& =\frac{1}{2} \int_{0}^{1} \frac{1}{\left(1-\rho r^{2}\right)^{2}} d r \cdot\|A\|_{L_{a}^{1}\left(D, \ell_{2}\right)} \cdot\|B\|_{\mathcal{B}\left(D, \ell_{2}\right)} \\
& =\frac{1}{2(1-\rho)} \cdot\|A\|_{L_{a}^{1}\left(D, \ell_{2}\right)} \cdot\|B\|_{\mathcal{B}\left(D, \ell_{2}\right)} .
\end{aligned}
$$

Therefore the series $\sum_{k=0}^{\infty} \rho^{k} r^{2 k}\left(\sum_{l=1}^{\infty} a_{k}^{l} \overline{b_{k}^{l}}\right)$ can be integrated term by term, and thus

$$
\langle A(\rho), B\rangle=\sum_{k=0}^{\infty} \frac{\rho^{k}}{k+1} \sum_{l=1}^{\infty} a_{k}^{l} \overline{b_{k}^{l}}, \quad \text { for all } 0 \leq \rho<1 .
$$

Now using (3.1) we find that

$$
|\langle A(\rho), B\rangle-\langle A, B\rangle| \leq\|A(\rho)-A\|_{L_{a}^{1}\left(D, \ell_{2}\right)} \cdot\|B\|_{\mathcal{B}\left(D, \ell_{2}\right)} \cdot
$$

We claim that

$$
\lim _{\rho \rightarrow 1^{-}}\|A(\rho)-A\|_{L_{a}^{1}\left(D, \ell_{2}\right)}=0
$$


Indeed, for $A \in L_{a}^{1}\left(D, \ell_{2}\right)$, the integral means $M_{1}(r, A)=\frac{1}{2 \pi} \int_{0}^{2 \pi}\left\|A\left(r e^{i \theta}\right)\right\|_{S_{1}} d \theta$, $0 \leq r<1$, are increasing functions of $r$, and, obviously,

Consequently,

$$
M_{1}(r, A(\rho))=M_{1}(r \rho, A) .
$$

$$
M_{1}(r, A-A(\rho)) \leq 2 M_{1}(r, A) .
$$

But it is easy to observe that $A \in L_{a}^{1}\left(D, \ell_{2}\right)$ if and only if $M_{1}(r, A) \in$ $L^{1}([0,1], r d r)$, and that $\|A(\rho z)-A(z)\|_{S_{1}} \rightarrow 0$ uniformly on the compact subsets of $D$, whenever $\rho \rightarrow 1^{-}$, which implies that

for all $r \in[0,1)$.

$$
\lim _{\rho \rightarrow 1^{-}} M_{1}(r, A-A(\rho))=0,
$$

Thus, by using the dominated convergence theorem, we obtain that

$$
\|A-A(\rho)\|_{L_{a}^{1}\left(D, \ell_{2}\right)}=2 \int_{0}^{1} M_{1}(r, A-A(\rho)) r d r \rightarrow 0,
$$

whenever $\rho \rightarrow 1^{-}$, i.e. (3.3) is proved.

From (3.2) and (3.3) we get that

$$
\lim _{\rho \rightarrow 1} \sum_{k=0}^{\infty} \frac{\rho^{k}}{k+1} \sum_{l=1}^{\infty} a_{k}^{l} \overline{b_{k}^{l}}=\lim _{\rho \rightarrow 1}\langle A(\rho), B\rangle=\langle A, B\rangle,
$$

for $A \in L_{a}^{1}\left(D, \ell_{2}\right)$ and $B \in \mathcal{B}\left(D, \ell_{2}\right)$. Consequently the condition (2) is satisfied for $A * B$.

It follows that $A * B \in \mathcal{A}^{\prime}\left(\ell_{2}\right)$, that is

$$
\mathcal{B}\left(D, \ell_{2}\right) \subset \mathcal{U T}\left(L_{a}^{1}\left(D, \ell_{2}\right), \mathcal{A}^{\prime}\left(\ell_{2}\right)\right)
$$

Conversely, let $\psi \in\left(L_{a}^{1}\left(D, \ell_{2}\right)\right)^{*}$. Then, by using the arguments in the proof of Theorem 7.11 in [18], it follows that there is an absolute constant $C>0$ and $B \in \mathcal{B}\left(D, \ell_{2}\right)$ such that

$$
\psi(A)=\langle A, B\rangle, \quad \text { for all } A \in L_{a}^{1}\left(D, \ell_{2}\right)
$$

and $\|\psi\| \geq C\|B\|_{\mathcal{B}\left(D, \ell_{2}\right)}$.

It follows that

$$
\left(L_{a}^{1}\left(D, \ell_{2}\right)\right)^{*} \subset \mathcal{B}\left(D, \ell_{2}\right) .
$$

Moreover,

$$
\mathcal{U T}\left(L_{a}^{1}\left(D, \ell_{2}\right), \mathcal{A}^{\prime}\left(\ell_{2}\right)\right) \subset\left(L_{a}^{1}\left(D, \ell_{2}\right)\right)^{*},
$$


360 Anca N. Marcoci, Liviu G. Marcoci, Lars-Erik Persson and Nicolae Popa

since if $A * B \in \mathcal{A}^{\prime}\left(\ell_{2}\right)$ for all $A \in L_{a}^{1}\left(D, \ell_{2}\right)$ then, by the previously remark, $\||B|\|<\infty$, and we can define $\psi \in\left[L_{a}^{1}\left(D, \ell_{2}\right)\right]^{*}$ as

and

$$
\psi(A)=\overline{\langle\bar{A}, B\rangle}=\lim _{\rho \rightarrow 1} \sum_{k=0}^{\infty} \frac{\rho^{k}}{k+1} \sum_{l=1}^{\infty} a_{k}^{l} b_{k}^{l}
$$

$$
\|\psi\|=\sup _{\|A\|_{L_{a}^{1}\left(D, \ell_{2}\right)} \leq 1}|\langle\bar{A}, B\rangle| \leq \sup _{\substack{0 \leq \rho<1 \\\|A\|_{L_{a}^{1}\left(D, \ell_{2}\right)}^{1} \leq 1}}\left|\sum_{k=0}^{\infty} \frac{\rho^{k}}{k+1} \sum_{l=1}^{\infty} a_{k}^{l} b_{k}^{l}\right| \leq\||B|\|<\infty .
$$

Consequently,

$$
\mathcal{U T}\left(L_{a}^{1}\left(D, \ell_{2}\right), \mathcal{A}^{\prime}\left(\ell_{2}\right)\right) \subset \mathcal{B}\left(D, \ell_{2}\right)
$$

The proof is complete by combining this embedding with (3.4).

We define

$$
\begin{aligned}
\mathcal{I}\left(\ell_{2}\right):=\{A \text { upper triangular matrices : } & \\
& \left.\|A\|_{\mathcal{I}\left(\ell_{2}\right)}:=\left\|A_{0}\right\|_{S_{1}}+\int_{0}^{1}\left\|A^{\prime}(r)\right\|_{S_{1}} 2 r d r<\infty\right\} .
\end{aligned}
$$

For the proof of our next result in this Section we need the following Lemma of independent interest:

Lemma 3.2. Let $\mathcal{I}\left(\ell_{2}\right)^{*}$ be the topological dual of $\mathcal{I}\left(\ell_{2}\right)$. Then

$$
\mathcal{I}\left(\ell_{2}\right)^{*}=\mathcal{B}\left(D, \ell_{2}\right)
$$

where the duality is given by

$$
\langle A, B\rangle=\lim _{\rho \rightarrow 1^{-}} \sum_{k=0}^{\infty} \rho^{k} \sum_{l=1}^{\infty} a_{k}^{l} \overline{b_{k}^{l}}
$$

for $A=\left(a_{k}^{l}\right)_{k \geq 0, l \geq 1} \in \mathcal{I}\left(\ell_{2}\right)$ and $B=\left(b_{k}^{l}\right)_{k \geq 0, l \geq 1} \in \mathcal{B}\left(D, \ell_{2}\right)$. The norms are equivalent.

Proof. Let $\psi \in \mathcal{I}\left(\ell_{2}\right)^{*}$. Since $\mathcal{I}\left(\ell_{2}\right)$ is the space of all upper triangular matrices $A$ such that $A^{\prime} \in L_{a}^{1}\left(D, \ell_{2}\right), \mathcal{I}\left(\ell_{2}\right)$ is a normed subspace of $L_{a}^{1}\left(D, \ell_{2}\right)$ and, we can use the Hahn-Banach Theorem to get an extension $\widetilde{\psi} \in L_{a}^{1}\left(D, \ell_{2}\right)^{*}$ such that

$$
\|\psi\|=\|\widetilde{\psi}\|
$$


Taking into account that $B \in \mathcal{B}\left(D, \ell_{2}\right)$ is the dual space of $L_{a}^{1}\left(D, \ell_{2}\right)$ (see e.g. Theorem 7.11 in [18]) there exists a unique $\widetilde{B} \in \mathcal{B}\left(D, \ell_{2}\right)$ such that

$$
\psi(A)=\widetilde{\psi}\left(A^{\prime}\right)=\lim _{\rho \rightarrow 1} \sum_{k=0}^{\infty} \frac{2 k \rho^{k}}{2 k+1} \sum_{l=1}^{\infty} a_{k}^{l} \tilde{b}_{k}^{\underline{l}} .
$$

Hence, taking

$$
B_{k}=\frac{2 k}{2 k+1} \widetilde{B_{k}}, \quad k=0,1,2, \ldots,
$$

it follows that $B \in \mathcal{B}\left(D, \ell_{2}\right)$,

$$
\|B\|_{\mathcal{B}\left(D, \ell_{2}\right)} \approx\|\widetilde{B}\|_{\mathcal{B}\left(D, \ell_{2}\right)} \approx\|\psi\|,
$$

and

$$
\psi(A)=\lim _{\rho \rightarrow 1^{-}} \sum_{k=0}^{\infty} \rho^{k} \sum_{l=1}^{\infty} a_{k}^{l} \overline{b_{k}^{l}}, \quad \text { for all } A \in \mathcal{I}\left(\ell_{2}\right) .
$$

Therefore

$$
\mathcal{I}\left(\ell_{2}\right)^{*} \subset \mathcal{B}\left(D, \ell_{2}\right)
$$

and

$$
\|\psi\|=\|\widetilde{\psi}\| \geq C\|B\|_{\mathcal{B}\left(D, \ell_{2}\right)} .
$$

Conversely, let $B \in \mathcal{B}\left(D, \ell_{2}\right)$. It defines a linear functional $\widetilde{\psi} \in L_{a}^{1}\left(D, \ell_{2}\right)^{*}$,

$$
\widetilde{\psi}(A)=\lim _{\rho \rightarrow 1^{-}} \sum_{k=0}^{\infty} \rho^{k} \sum_{l=1}^{\infty} a_{k}^{l} \overline{b_{k}^{l}}
$$

such that $\|\widetilde{\psi}\| \approx\|B\|_{\mathcal{B}\left(D, \ell_{2}\right)}$.

Consequently, taking $\psi=\left.\widetilde{\psi}\right|_{\mathcal{I}\left(\ell_{2}\right)}$, we obtain that

$$
\|\psi\| \leq C_{2}\|B\|_{\mathcal{B}\left(D, \ell_{2}\right)}
$$

and $\mathcal{B}\left(D, \ell_{2}\right) \subset \mathcal{I}\left(\ell_{2}\right)^{*}$. The proof is complete.

Our next result shows that the Bloch space of upper triangular infinite matrices can be regarded as the space of Schur multipliers between the predual of the Bloch space and the space of all Abel summable matrices, $\mathcal{A}\left(\ell_{2}\right)$ defined as all upper triangular matrices $A$ with absolutely summable diagonals such that

$$
\sup _{k \geq 0} \sum_{l=1}^{\infty}\left|a_{k}^{l}\right|<\infty, \lim _{r \rightarrow 1^{-}} \sum_{k=0}^{\infty} r^{k} \sum_{l=1}^{\infty} a_{k}^{l}
$$


362 Anca N. Marcoci, Liviu G. Marcoci, Lars-Erik Persson and Nicolae Popa

exists, and similarly as in the previously remark it follows that

$$
\sup _{\substack{0 \leq r<1 \\\|B\|_{\mathcal{I}\left(\ell_{2}\right) \leq 1}}}\left|\sum_{k=0}^{\infty} r^{k} \sum_{l=1}^{\infty} a_{k}^{l} b_{k}^{l}\right|<\infty .
$$

We are now ready to formulate our next result.

Theorem 3.3. It yields that

as linear spaces.

$$
\mathcal{B}\left(D, \ell_{2}\right)=\mathcal{U T}\left(\mathcal{I}\left(\ell_{2}\right), \mathcal{A}\left(\ell_{2}\right)\right)
$$

Proof. We consider first $A \in \mathcal{U T}\left(\mathcal{I}\left(\ell_{2}\right), \mathcal{A}\left(\ell_{2}\right)\right)$, which implies that

$$
A * B \in \mathcal{A}\left(\ell_{2}\right),
$$

for every $B \in \mathcal{I}\left(\ell_{2}\right)$, i.e. the limit

$$
\lim _{r \rightarrow 1^{-}} \sum_{k=0}^{\infty} r^{k} \sum_{l=1}^{\infty} a_{k}^{l} b_{k}^{l}
$$

exists.

We denote by $\psi(B)$ the previous limit. Then we have that

$$
\sup _{\|B\|_{\mathcal{I}\left(\ell_{2}\right)} \leq 1}|\psi(B)| \leq\|A\|_{\left(\mathcal{I}\left(\ell_{2}\right), \mathcal{A}\left(\ell_{2}\right)\right)}<\infty,
$$

which in its turn implies that $\psi \in\left(\mathcal{I}\left(\ell_{2}\right)\right)^{*}$. By using Lemma 3.2 and that

$$
\langle A, \bar{B}\rangle=\psi(B), \quad \text { for every } B \in \mathcal{I}\left(\ell_{2}\right),
$$

it follows that $A \in \mathcal{B}\left(D, \ell_{2}\right)$.

Therefore we obtain that

$$
\mathcal{U} \mathcal{T}\left(\mathcal{I}\left(\ell_{2}\right), \mathcal{A}\left(\ell_{2}\right)\right) \subset \mathcal{B}\left(D, \ell_{2}\right) .
$$

Conversely, if $A \in \mathcal{B}\left(D, \ell_{2}\right)$ and $B \in \mathcal{I}\left(\ell_{2}\right)$, by Lemma 3.2 (see also the proof of Lemma 7.7 in [1]) there exists

$$
\lim _{r \rightarrow 1^{-}} \sum_{k=0}^{\infty} r^{k}\left(\sum_{l=1}^{\infty} a_{k}^{l} b_{k}^{l}\right)
$$

and

$$
\sup _{k} \sum_{l=1}^{\infty}\left|a_{k}^{l}\right|\left|b_{k}^{l}\right| \leq\|A\|_{\mathcal{B}\left(D, \ell_{2}\right)} \cdot\|B\|_{\mathcal{I}\left(\ell_{2}\right)}<\infty .
$$

Thus $A * B \in \mathcal{A}\left(\ell_{2}\right)$, that is

$$
\mathcal{B}\left(D, \ell_{2}\right) \subset \mathcal{U} \mathcal{T}\left(\mathcal{I}\left(\ell_{2}\right), \mathcal{A}\left(\ell_{2}\right)\right) .
$$

The proof is complete. 
In [19] (see also [18]) was introduced and studied the closed Banach subspace $\mathcal{B}_{0, c}\left(D, \ell_{2}\right)$ of the little Bloch space $\mathcal{B}_{0}\left(D, \ell_{2}\right)$. This space consists in all upper triangular matrices whose diagonals are compact operators. Our next result shows that the space $\mathcal{B}_{0, c}\left(D, \ell_{2}\right)$ is the predual of the space $\mathcal{I}\left(\ell_{2}\right)$. More precisely we have:

Theorem 3.4. It yields that $\mathcal{B}_{0, c}\left(D, \ell_{2}\right)^{*}=\mathcal{I}\left(\ell_{2}\right)$ with equivalence of norms.

Proof. We use some ideas from the proof of Theorem 2.4 in [1]. By the Hahn-Banach theorem it follows that

$$
\|G\|_{\mathcal{I}\left(\ell_{2}\right)}=\sup _{\Phi \neq 0} \frac{|\Phi(G)|}{\|\Phi\|_{\mathcal{I}\left(\ell_{2}\right)^{*}}} .
$$

Then, by using Lemma 3.2, we get that

$$
C_{1}\|G\|_{\mathcal{I}\left(\ell_{2}\right)} \leq \sup _{F \neq 0} \frac{|\langle F, G\rangle|}{\|F\|_{\mathcal{B}\left(D, \ell_{2}\right)}} \leq C_{2}\|G\|_{\mathcal{I}\left(\ell_{2}\right)}
$$

where

$$
\langle F, G\rangle=\lim _{\rho \rightarrow 1^{-}} \sum_{k=0}^{\infty} \rho^{k} \sum_{l=1}^{\infty} f_{k}^{l} \cdot \overline{g_{k}^{l}},
$$

$F=\left(f_{k}^{l}\right)_{k \geq 0, l \geq 1}$ and $G=\left(g_{k}^{l}\right)_{k \geq 0, l \geq 1}$.

Let $\Phi \in \mathcal{B}_{0, c}\left(D, \ell_{2}\right)^{*}$ be given and let $E_{k}^{l}, k \geq 0, l \geq 1$, be the matrix having the entry 1 on the $l^{\text {th }}$ place on the $k^{\text {th }}$ diagonal, and 0 otherwise, i.e. $E_{k}^{l}=\left(e_{i}^{j}\right)_{i \geq 0, j \geq 1}$, where

$$
e_{i}^{j}= \begin{cases}1, & \text { if } i=k \text { and } j=l \\ 0, & \text { otherwise }\end{cases}
$$

We define $b_{k}^{l}:=\overline{\Phi\left(E_{k}^{l}\right)}, k \geq 0, l \geq 1$, and for fixed $k \geq 0, B_{k}:=\left(b_{k}^{l}\right)_{l \geq 1}$. Then $G=\sum_{k=0}^{\infty} B_{k}$, the series being a formal one.

First we show that $\left(b_{k}^{l}\right)_{l \geq 1}$ is an absolutely summing sequence for every $k \geq 0$.

For fixed $n \geq 1$, and $k \geq 0$, we have that

$$
\begin{aligned}
\left|\Phi\left(\sum_{l=1}^{n} f_{k}^{l} E_{k}^{l}\right)\right|=\left|\sum_{l=1}^{n} f_{k}^{l} \overline{b_{k}^{l}}\right| & \leq\|\Phi\|_{\mathcal{B}_{0, c}\left(D, \ell_{2}\right)^{*}} \cdot\left\|\sum_{l=1}^{n} f_{k}^{l} E_{k}^{l}\right\|_{\mathcal{B}_{0, c}\left(D, \ell_{2}\right)} \\
& \leq\|\Phi\|_{\mathcal{B}_{0, c}\left(D, \ell_{2}\right)^{*}} \cdot\left\|F_{k}\right\|_{\mathcal{B}_{0, c}\left(D, \ell_{2}\right)}
\end{aligned}
$$

where $F_{k}=\left(f_{k}^{l}\right)_{l \geq 1}$ and $F=\left(f_{k}^{l}\right)_{k \geq 0, l \geq 1} \in \mathcal{B}_{0, c}\left(D, \ell_{2}\right)$. 
364 Anca N. Marcoci, Liviu G. Marcoci, Lars-Erik Persson and Nicolae Popa

Since

$$
\sup _{l \geq 1}\left|f_{k}^{l}\right| \sim\left\|F_{k}\right\|_{\mathcal{B}_{0, c}\left(D, \ell_{2}\right)} \leq C\|F\|_{\mathcal{B}_{0, c}\left(D, \ell_{2}\right)},
$$

for all $k \geq 0$ and for all $F \in \mathcal{B}_{0, c}\left(D, \ell_{2}\right)$, it follows that

$$
\sum_{l=1}^{n}\left|b_{k}^{l}\right|=\sup _{\sup _{l \geq 1}\left|f_{k}^{l}\right| \leq 1}\left|\sum_{l=1}^{n} f_{k}^{l} b_{k}^{l}\right| \leq C\|\Phi\|_{\mathcal{B}_{0, c}\left(D, \ell_{2}\right)^{*}},
$$

where $C>0$ is an absolute constant.

Consequently,

$$
\sum_{l=1}^{\infty}\left|b_{k}^{l}\right| \leq C\|\Phi\|_{\mathcal{B}_{0, c}\left(D, \ell_{2}\right)^{*}}
$$

for all $k \geq 0$.

Next we show that

$$
\Phi(F)=\langle F, G\rangle=\lim _{\rho \rightarrow 1^{-}} \sum_{k=0}^{\infty} \rho^{k} \sum_{l=1}^{\infty} f_{k}^{l} \overline{b_{k}^{l}},
$$

for every $F \in \mathcal{B}_{0, c}\left(D, \ell_{2}\right)$.

Let $0 \leq r<1$ and $F_{r}=\sum_{k=0}^{\infty} r^{k} F_{k}$. Since $\left\|F_{k}\right\|_{\mathcal{B}_{0, c}\left(D, \ell_{2}\right)} \leq\|F\|_{\mathcal{B}_{0, c}\left(D, \ell_{2}\right)}$ for every $k \geq 0$, the previous series is absolutely summing in $\mathcal{B}_{0, c}\left(D, \ell_{2}\right)$.

We also notice that

$$
\Phi\left(F_{k}\right)=\sum_{l=1}^{\infty} f_{k}^{l} \Phi\left(E_{k}^{l}\right)=\sum_{l=1}^{\infty} f_{k}^{l} \overline{b_{k}^{l}}
$$

the last series being summable since $\left(f_{k}^{l}\right)_{l=1}^{\infty} \in c_{0}$, and $\left(b_{k}^{l}\right)_{l=1}^{\infty} \in \ell_{1}$, for every $k \geq 0$.

Hence,

$$
\Phi\left(F_{r}\right)=\sum_{k=0}^{\infty} r^{k} \Phi\left(F_{k}\right)=\sum_{k=0}^{\infty} r^{k} \sum_{l=1}^{\infty} f_{k}^{l} \overline{b_{k}^{l}}
$$

and

$$
\left|\Phi\left(F_{r}\right)\right| \leq \sum_{k=0}^{\infty} r^{k} \sum_{l=1}^{\infty}\left|f_{k}^{l}\right|\left|b_{k}^{l}\right| \leq \frac{C}{1-r}\|F\|_{\mathcal{B}_{0, c}\left(D, \ell_{2}\right)} \cdot\|\Phi\|_{\mathcal{B}_{0, c}\left(D, \ell_{2}\right)^{*}}
$$

Since $F_{r} \rightarrow F$ in $\mathcal{B}_{0, c}\left(D, \ell_{2}\right)$, as $r \rightarrow 1^{-}$, (see [18], p. 162) it follows that

$$
\Phi(F)=\lim _{r \rightarrow 1^{-}} \Phi\left(F_{r}\right)=\lim _{r \rightarrow 1^{-}} \sum_{k=0}^{\infty} r^{k} \sum_{l=1}^{\infty} f_{k}^{l} \overline{b_{k}^{l}}=\langle F, G\rangle,
$$


for all $F \in \mathcal{B}_{0, c}\left(D, \ell_{2}\right)$.

Now, let $0<\rho<1$. We observe that

and

$$
G_{\rho}=\sum_{n=0}^{\infty} \rho^{n} B_{n} \in \mathcal{I}\left(\ell_{2}\right),
$$

$$
\left\|B_{n}\right\|_{\mathcal{I}\left(\ell_{2}\right)} \leq C\|\Phi\|_{\mathcal{B}_{0, c}\left(D, \ell_{2}\right)^{*}},
$$

for every $n \geq 0$.

If $F \in \mathcal{B}_{0, c}\left(D, \ell_{2}\right)$, then, by using (3.5), we find that

$$
\begin{aligned}
\left|\left\langle F, G_{\rho}\right\rangle\right| & =\left|\left\langle F_{\rho}, G\right\rangle\right|=\left|\Phi\left(F_{\rho}\right)\right| \leq\|\Phi\|_{\mathcal{B}_{0, c}\left(D, \ell_{2}\right)^{*}} \cdot\left\|F_{\rho}\right\|_{\mathcal{B}_{0, c}\left(D, \ell_{2}\right)} \\
& \leq\|\Phi\|_{\mathcal{B}_{0, c}\left(D, \ell_{2}\right)^{*}} \cdot\|F\|_{\mathcal{B}_{0, c}\left(D, \ell_{2}\right)} .
\end{aligned}
$$

From Lemma 3.2 we have that

$$
\left\|G_{\rho}\right\|_{\mathcal{I}\left(\ell_{2}\right)} \leq C_{1}^{-1} \sup _{F \neq 0} \frac{\left|\left\langle F, G_{\rho}\right\rangle\right|}{\|F\|_{\mathcal{B}_{0, c}\left(D, \ell_{2}\right)}} \leq C_{1}^{-1} \cdot\|\Phi\|_{\mathcal{B}_{0, c}\left(D, \ell_{2}\right)^{*}} .
$$

Hence, $G \in \mathcal{I}\left(\ell_{2}\right)$, and

$$
\|G\|_{\mathcal{I}\left(\ell_{2}\right)} \leq C_{1}^{-1} \cdot\|\Phi\|_{\mathcal{B}_{0, c}\left(D, \ell_{2}\right)^{*}} .
$$

We conclude that $\mathcal{B}_{0, c}\left(D, \ell_{2}\right)^{*} \subset \mathcal{I}\left(\ell_{2}\right)$. The converse inclusion follows in the same way as in the last part of the proof of Lemma 3.2, so we omit the details. The proof is complete.

In our last result of this Section we prove that the space of all upper triangular Schur multipliers from the space little Bloch whose diagonals are compact operators into the space of Abel summable matrices is exactly the space $\mathcal{I}\left(\ell_{2}\right)$.

Theorem 3.5. It yields that

as linear spaces.

$$
\mathcal{I}\left(\ell_{2}\right)=\mathcal{U} \mathcal{T}\left(\mathcal{B}_{0, c}\left(D, \ell_{2}\right), \mathcal{A}\left(\ell_{2}\right)\right)
$$

Proof. If $A \in \mathcal{I}\left(\ell_{2}\right)$ and $B \in \mathcal{B}_{0, c}\left(D, \ell_{2}\right)$, it follows that $A * B \in \mathcal{A}\left(\ell_{2}\right)$ by the proof of Theorem 3.3.

Hence,

$$
\mathcal{I}\left(\ell_{2}\right) \subset \mathcal{U T}\left(\mathcal{B}_{0, c}\left(D, \ell_{2}\right), \mathcal{A}\left(\ell_{2}\right)\right) .
$$

Conversely, let $A \in \mathcal{U T}\left(\mathcal{B}_{0, c}\left(D, \ell_{2}\right), \mathcal{A}\left(\ell_{2}\right)\right)$. Then, for $B \in \mathcal{B}_{0, c}\left(D, \ell_{2}\right)$ we have that $A * B \in \mathcal{A}\left(\ell_{2}\right)$, that is

$$
\langle A, B\rangle=\lim _{r \rightarrow 1^{-}} \sum_{k=0}^{\infty} r^{k}\left(\sum_{l=1}^{\infty} a_{k}^{l} b_{k}^{l}\right)
$$


366 Anca N. Marcoci, Liviu G. Marcoci, Lars-Erik Persson and Nicolae Popa

exists. Let us denote $\psi(B)=\lim _{r \rightarrow 1^{-}} \sum_{k=0}^{\infty} r^{k}\left(\sum_{l=1}^{\infty} a_{k}^{l} b_{k}^{l}\right)$. Then it follows that

$$
\sup _{\|B\|_{\mathcal{B}_{0, c}\left(D, \ell_{2}\right)} \leq 1}|\psi(B)| \leq\|A\|_{\left(\mathcal{B}_{0, c}\left(D, \ell_{2}\right), \mathcal{A}\left(\ell_{2}\right)\right)}<\infty .
$$

Since $\psi \in \mathcal{B}_{0, c}\left(D, \ell_{2}\right)^{*}$ and $\langle A, B\rangle=\psi(B)$, from Theorem 3.4 we obtain that $A \in \mathcal{I}\left(\ell_{2}\right)$. Therefore

$$
\mathcal{U T}\left(\mathcal{B}_{0, c}\left(D, \ell_{2}\right), \mathcal{A}\left(\ell_{2}\right)\right) \subset \mathcal{I}\left(\ell_{2}\right)
$$

and the proof is complete.

\section{Applications}

In this last Section we give some applications of our results.

For a space of upper triangular infinite matrices $X$ we denote the Abel dual by $X^{a}=\mathcal{U T}\left(X, \mathcal{A}\left(\ell_{2}\right)\right)$, where $\mathcal{A}\left(\ell_{2}\right)$ is the space of all Abel summable matrices. We also denote the Köthe dual by $X^{K}=\mathcal{U} \mathcal{T}\left(X, \mathcal{M}_{1}\right)$. It is clear that $X^{K} \subset X^{a}$.

More generally, we denote by $X^{H}=\mathcal{U} \mathcal{T}(X, H)$, where $H$ is a fixed space of infinite matrices.

It is known that in [2] is obtained that the largest solid subspace of the Abel dual of a sequence space coincides with the Köthe dual of the same sequence space. The extension in the case of infinite matrices is the following:

Proposition 4.1. Let $X$ or $Y$ be two spaces of analytic matrices. Then we have $s\left(X^{a}\right)=X^{K}$.

Proof. Let us observe first that $\mathcal{U T}(X, Y) \subset \mathcal{U} \mathcal{T}\left(Y^{H}, X^{H}\right)$. Since $X^{H H H}=$ $X^{H}$ it follows that $\mathcal{U} \mathcal{T}\left(X^{H}, Y^{H}\right)=\mathcal{U} \mathcal{T}\left(Y^{H H}, X^{H H}\right)$. From Proposition 2.2 we have that

$$
s\left(X^{a}\right)=\mathcal{U} \mathcal{T}\left(\mathcal{M}_{\infty}, X^{a}\right)=\mathcal{U} \mathcal{T}\left(X^{a a}, \mathcal{M}_{1}\right) \subset \mathcal{U T}\left(X, \mathcal{M}_{1}\right)=X^{K} .
$$

Conversely, $X^{K}$ is solid and $X^{K} \subset X^{a}$ so that

$$
X^{K} \subset s\left(X^{a}\right) \text {. }
$$

The proof is complete.

Remark 4.2. If $X$ is solid, then

$$
X^{a}=X^{K}
$$

while in the general case we only have

$$
X^{K} \subset X^{a}
$$


Our next result describes the largest solid subspace of Schur multipliers between two spaces of infinite matrices.

Proposition 4.3. Let $X$ and $Y$ be two spaces of analytic matrices. Then

$$
(X, s(Y))=s((X, Y))
$$

Proof. Let $A$ be in $(X, s(Y))$. In view of Proposition 2.2 this is equivalent to that

$$
A * B * C \in Y,
$$

for every $B \in X$ and for every $C \in \mathcal{M}_{\infty}$ i.e.

$$
A * C \in(X, Y) \text {, for every } C \in \mathcal{M}_{\infty},
$$

which in its turn means that $A \in s((X, Y))$.

Finally, as applications of our previous results, we are able to give a complete description of the largest solid subspace of $\mathcal{B}\left(D, \ell_{2}\right)$ and $\mathcal{I}\left(\ell_{2}\right)$ in terms of the Köthe dual. Moreover, for an arbitrary space of infinite matrices $X$, the Schur multipliers from $X$ to the largest solid subspace of $\mathcal{B}\left(D, \ell_{2}\right)$ (or $\mathcal{I}\left(\ell_{2}\right)$ ) is exactly the largest solid subspace of the solid space of multipliers from $X$ to $\mathcal{B}\left(D, \ell_{2}\right)$ (respectively $\mathcal{I}\left(\ell_{2}\right)$ ).

Theorem 4.4. The following identities hold:

(i) $s\left(\mathcal{B}\left(D, \ell_{2}\right)\right)=\mathcal{I}\left(\ell_{2}\right)^{K}$ with equivalence of norms;

(ii) $s\left(\mathcal{I}\left(\ell_{2}\right)\right)=\mathcal{B}_{0, c}\left(D, \ell_{2}\right)^{K}$ with equivalence of norms.

Proof. Indeed, from Theorem 3.3 we have that $\mathcal{B}\left(D, \ell_{2}\right)=\mathcal{I}\left(\ell_{2}\right)^{a}$, and therefore, by applying Proposition 4.1, we find that the identity (i) holds.

The statement (ii) follows from Theorem 3.5 and Proposition 4.1.

Corollary 4.5. Let $X$ be a space of analytic matrices. Then

(a) $\mathcal{U T}\left(X, s\left(\mathcal{B}\left(D, \ell_{2}\right)\right)\right)=\mathcal{U T}\left(X, \mathcal{I}\left(\ell_{2}\right)^{K}\right)=s\left(\mathcal{U T}\left(X, \mathcal{B}\left(D, \ell_{2}\right)\right)\right)$;

(b) $\mathcal{U} \mathcal{T}\left(X, s\left(\mathcal{I}\left(\ell_{2}\right)\right)\right)=\mathcal{U} \mathcal{T}\left(X, \mathcal{B}_{0, c}\left(D, \ell_{2}\right)^{K}\right)=s\left(\mathcal{U T}\left(X, \mathcal{I}\left(\ell_{2}\right)\right)\right)$.

Proof. The first equality in (a) follows from Theorem 4.4 (i) and applying Proposition 4.3 we obtain the second one.

The equalities in (b) follows from Proposition 4.3 and Theorem 4.4 (ii). 
Conjecture. Influenced by [2] we conjecture that

$$
\mathcal{B}\left(D, \ell_{2}\right)^{K}=\mathcal{M}(2,1)
$$

where $\mathcal{M}(2,1)$ is the space of all upper triangular matrices $A=\left(a_{k}^{l}\right)_{k \geq 0, l \geq 1}$ such that

$$
\left\{\left(\sum_{k \in I_{n}}\left\|A_{k}\right\|^{2}\right)^{1 / 2}\right\}_{n=0}^{\infty} \in \ell_{1},
$$

where $\left\|A_{k}\right\|_{\infty}=\sup _{l \geq 1}\left|a_{k}^{l}\right|$ and $I_{n}=\left\{k: 2^{n} \leq k<2^{n+1}\right\},(n=0,1, \ldots)$.

Final remark. In the literature there are some papers studying the notion of pointwise domination property, which is obviously related to the present notion of a solid space.

A matrix space $X$ has the pointwise domination property, if $\left|a_{i j}\right| \leq b_{i j}$ for all $i, j \in \mathbb{N}$ and $B=\left(b_{i j}\right) \in X$ implies $A=\left(a_{i j}\right) \in X$. This property has been investigated by B. Simon in his monograph [20] in the context of Schatten classes. Clearly $S_{2}$ has this property, and from this he derived that $S_{p}$ has the pointwise domination property if $p$ is an even integer (Theorem 2.13). Moreover, V. V. PELLER [17] proved that all other $S_{p}$, when $p$ is not an even integer, fail to have this property. Subsequently the domination property was further studied, see e.g. [9]. The case of Lorentz-Schatten classes was considered in [8].

It seems to be interesting that although the domination property looks very closely related to the definition of solid matrix spaces, it leads to completely different results (for instance for Schatten classes) and requires other techniques.

ACKNOWLEDGEMENTS. The first named author was partially supported by POSDRU/159/1.5/S/137750. We thank the referees for very careful reading of the paper and for their valuable comments and suggestions. In particular, one of the referees pointed out the important information in our final remark.

\section{References}

[1] J. M. Anderson, J. Clunie and Ch. Pommerenke, On Bloch function and normal functions, J. Reine Angew. Math. 270 (1974), 12-37.

[2] J. M. Anderson and A. Shields, Coefficient multipliers of Bloch functions, Trans. Amer. Math. Soc. 224 (1976), 255-265.

[3] J. L. Arregui and O. Blasco, Bergman and Bloch spaces of vector-valued functions, Math. Nach. 261/262 (2003), 3-22.

[4] S. Barza, L. E. Persson and N. Popa, A matriceal analogue of Fejer's theory, Math. Nach. 260 (2003), 14-20. 
[5] S. Barza, V. D. Lie and N. Popa, Approximation of infinite matrices by matriceal Haar polynomials, Ark. Math. 43 (2005), 251-269.

[6] O. Blasco, On coefficient of vector-valued Bloch functions, Studia Math. 165 (2004), 101-110.

[7] O. Blasco, Introduction to vector valued Bergman spaces, function spaces and operator theory, 9-30, Univ. Joensuu Dept. Math. Rep. Ser., 8, Joensuu, 2005.

[8] F. Совоs and T. Küнn, Lorentz-Schatten classes and pointwise domination of matrices, Canad. Math. Bull. 42 (1999), 162-168.

[9] M. Dechamps-Gondim, F. Lust-Piquard and H. Queffelec, On the minorant properties in $C_{p}(H)$, Pacific J. Math. 119 (1985), 89-101.

[10] P. L. Duren, Theory of $H^{p}$ Spaces, Academic Press, New York, 1970.

[11] S. V. Kislyakov, Fourier coefficients of boundary values of analytic functions in the disc and bidisc, Spectral theory of functions and operators, II., Trudy Math. Inst. Steklov 155 (1981), 77-94, 183-185 (in Russian).

[12] M. Jevtić and M. Pavlović, On the solid hull of the Hardy space $H^{p}, 0<p<1$, Michigan Math. J. 54 (2006), 439-446.

[13] F. Lust-Piquard, On the coefficient problem: a version of the Kahane Katznelson de Leeuw theorem for spaces of matrices, J. Funct. Anal. 149 (1997), 352-376.

[14] L. G. Marcoci, L. E. Persson, I. Popa and N. Popa, A new characterization for a Bergman-Schatten spaces and a duality result, J. Math. Anal. Appl. 360 (2009), 67-80.

[15] M. Mateljević and M. PAVLović, $L_{p}$ behaviour of the integral means of analytic functions, Studia Math. 77 (1984), 219-237.

[16] A. Pelczynski and F. Sukochev, Some remarks on Toeplitz multipliers and Hankel matrices, Studia Math. 175 (2006), 175-204.

[17] V. V. PelLer, Hankel operators of class $S_{p}$ and their applications, Math. USSR Sb. 41 (1982), 443-479.

[18] L. E. Persson and N. Popa, Matrix Spaces and Schur Multipliers: Matriceal Harmonic Analysis, World Scientific, 2014.

[19] N. PopA, Matriceal Bloch and Bergman-Schatten spaces, Rev. Roumaine Math. Pures Appl. 52 (2007), 459-478.

[20] B. Simon, Trace Ideals and Their Applications, LMS Lecture Note Series 35, Cambridge University Press, Cambridge - New York, 1979. 
370 A. N. Marcoci et al. : Some new characterizations of Bloch type spaces ...

[21] K. ZHu, Operator Theory in Banach Function Spaces, Marcel Dekker, New York, 1990.

ANCA N. MARCOCI

DEPARTMENT OF MATHEMATICS

AND COMPUTER SCIENCE

TECHNICAL UNIVERSITY

OF CIVIL ENGINEERING BUCHAREST

RO-020396, BUCHAREST

ROMANIA

E-mail: anca.marcoci@utcb.ro

LARS-ERIK PERSSON

DEPARTMENT OF ENGINEERING

SCIENCES AND MATHEMATICS

LULEÅUNIVERSITY OF TECHNOLOGY

SE-97 187, LULE $\AA$

SWEDEN

AND

NARVIK UNIVERSITY COLLEGE

P.O. BOX $385, \mathrm{~N}-8505$ NARVIK

NORWAY

E-mail: larserik@ltu.se
LIVIU G. MARCOCI

DEPARTMENT OF MATHEMATICS

AND COMPUTER SCIENCE

TECHNICAL UNIVERSITY

OF CIVIL ENGINEERING BUCHAREST

RO-020396, BUCHAREST

ROMANIA

E-mail: lmarcoci@instal.utcb.ro

NICOLAE POPA

INSTITUTE OF MATHEMATICS

OF ROMANIAN ACADEMY

RO-014700 BUCHAREST, P.O. BOX 1-764

ROMANIA

E-mail: npopa@imar.ro

(Received October 13, 2014; revised March 21, 2015) 